\title{
Erratum: Saddle point anomaly of Landau levels in graphenelike structures [Phys. Rev. B 104, 035419 (2021)]
}

\author{
A. V. Nikolaev (1) \\ (Received 21 December 2021; published 6 January 2022)
}

DOI: 10.1103/PhysRevB.105.039902

In my original paper Landau levels have been studied in graphenelike structures and it was concluded that at the immediate vicinity of the saddle point Landau levels are broadened in minibands of finite bandwidth even in relatively small rational magnetic fields with the magnetic flux $f=p / q$, where $p$ and $q$ are coprime integers. In particular, the dispersion relations of such minibands are reproduced in Figs. 6 and 8 of my original paper for $q=1502$ and $q=1503$, respectively ( $p=1$ in both cases).

Although technically all data based on exact numerical calculations are correct, they are not representative because of the scale of these plots: the magnetic unit cell used for these calculations and shown in Fig. 3 of my original paper, is too big. The symmetry points $M_{x}, M_{y}$, and $M_{x y}$ used there are given by $\left(\pi / d_{x}, 0\right),\left(0, \pi / 2 d_{y}\right)$, and $\left(\pi / d_{x}, \pi / 2 d_{y}\right)$, correspondingly, where $d_{x}=3 a / 2$ and $d_{y}=a \sqrt{3} / 2$ ( $a$ is the C-C bond length in graphene). In real space the difference equations [1] (i.e., along the $x$ axis) are periodic with the shortest translation vector $2 q d_{x}$. This implies the shortest translation vector $2 \pi / 2 q d_{x}=\pi / q d_{x}$ along the $k_{x}$ direction in the reciprocal space. Analogously, one can show that the difference equations [1] have the shortest period $\pi / q d_{y}$ along the $k_{y}$ direction. The primitive magnetic unit cell then comprises a very small region in $k$ space (especially when $q \rightarrow \infty):-\pi / 2 q d_{x} \leqslant k_{x} \leqslant \pi / 2 q d_{x}$ and $-\pi / 2 q d_{y} \leqslant k_{y} \leqslant \pi / 2 q d_{y}$. Introducing new corner points of this rectangular primitive unit cell, $M_{x}^{\prime}\left(\pi / 2 q d_{x}, 0\right), M_{y}^{\prime}\left(0, \pi / 2 q d_{y}\right)$, and $M_{x y}^{\prime}\left(\pi / 2 q d_{x}, \pi / 2 q d_{y}\right)$, one can plot the dispersion dependencies along the high symmetry lines $\Gamma-M_{y}^{\prime}-M_{x y}^{\prime}-\Gamma-M_{x}^{\prime}-M_{x y}^{\prime}$, shown here in Figs. 1 and 2 . Now the length of each high symmetry line is of the order of $q \sim 10^{3}$ times smaller than the line lengths used in Figs. 6 and 8, and the reduced curves are explicitly smooth in contrast to those in Figs. 6 and 8 of my original paper, where the dispersion oscillations are so fast that the magnetic band structure shown here in Figs. 1 and 2 is hidden.

It should be mentioned that all other plots and conclusions in my original paper, including the density of plots and bandwidths, are correct.

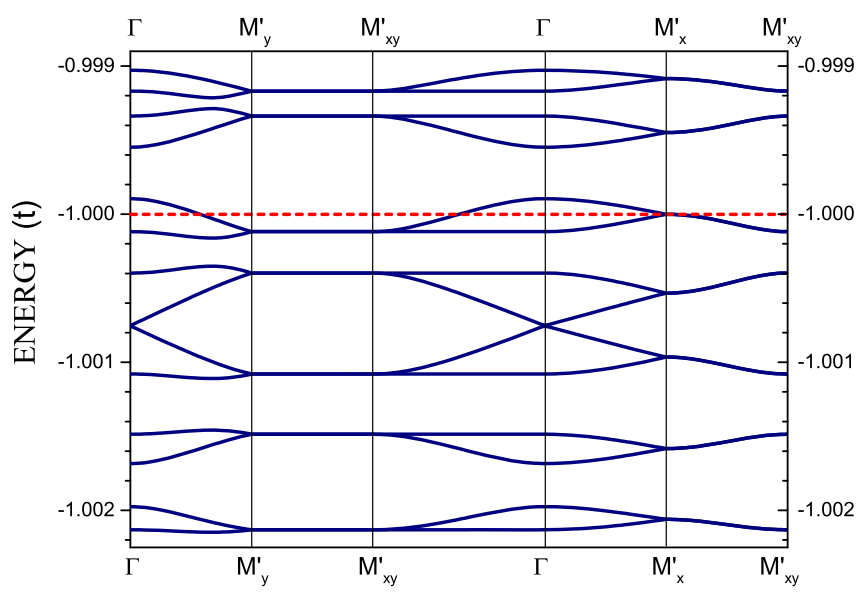

FIG. 1. Calculated magnetic band dispersions in the immediate vicinity of the saddle point, $H=52.6 \mathrm{~T}(q=1502, p=1)$ for high symmetry lines of the primitive magnetic unit cell. The total number of $k$ points is 201 . The saddle point energy $\left(\epsilon_{M}=-t\right)$ is shown by the red line. 


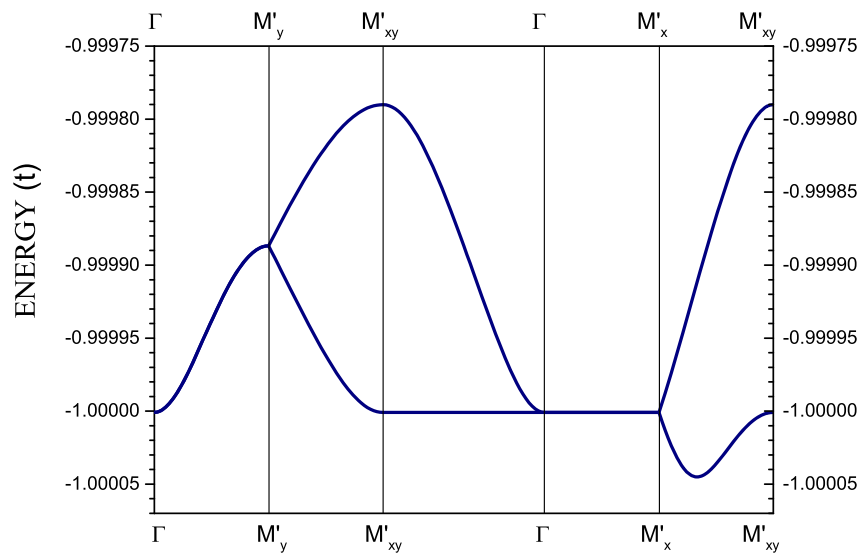

FIG. 2. Calculated dispersion law of the saddle point magnetic band for $q=1503, p=1(H=52.52 \mathrm{~T})$ for high symmetry lines of the primitive magnetic unit cell. The total number of $k$ points is 201 .

[1] R. Rammal, J. Phys. France 46, 1345 (1985). 